\title{
O-Alkylation of Chitosan for Gene Delivery by Using Ionic Liquid in an in- situ Reactor
}

\author{
Huiying Chen ${ }^{1}$, Shaohui Cui ${ }^{1}$, Yinan Zhao ${ }^{1}$, Bing Wang ${ }^{1}$, Shubiao Zhang ${ }^{1}$, Huiying Chen ${ }^{2}$, Xiaojun Peng ${ }^{2}$ \\ ${ }^{1}$ Key Laboratory of Bio-chemistry Engineering - The State Ethnic Affairs Commission-Ministry of Education, \\ Dalian Nationalities University, Dalian, China \\ ${ }^{2}$ State Key Laboratory of Fine Chemicals, Dalian University of Technology, Dalian, China \\ Email: zsb@dlnu.edu.cn
}

Received 2012

\begin{abstract}
An in-situ reactor was elaborately designed for O-alkylation of chitosan in an ionic liquid ([BMIM]Cl) solvent, using $\mathrm{N}$, N'-carbonyldiimidazole as bonding agent. The original chitosan and the modified chitosan were characterized by FT-IR and XRD analysis. FT-IR spectra revealed that the alkylation of chitosan selectively occurred at hydroxyl groups, with unprotected amino groups untouched. It was proposed that the particular properties of the ionic liquid solvent should be responsible for the selectively alkylation. The result from X-ray diffraction showed that the crystallinity of O-alkylation of chitosan decreases, most likely due to the decomposition of CS in the ionic liquid. The solubility test of O-alkylated chitosan in aqueous HAc solution (w/w: 0.1\%) confirmed that the product could be easily dissolved in aqueous HAc solution because of its abundant free amino groups. It was suggested that the O-alkylated chitosan was suitable for the coming cell transfection test in vitro.
\end{abstract}

Keywords: Gene Delivery; Chitosan; Alkylation; Ionic Liquid; Iu-Situ Reactor

\section{Introduction}

Gene delivery into mammalian cells has become an indispensable research tool in molecular and cell biology [1]. Despite widespread use and numerous delivery systems available, transfection is still a matter of compromise between acceptable toxicity and efficacy [2, 3]. Compared with viral vectors, nonviral carriers are considerably safer and easy to produce, and they possess large gene-carrying capacity and flexibility of design [4]. Because lack of toxicity is a major demand in the design of new gene delivery systems, the development of nonviral vectors has been increasingly focused on biocompatible systems and natural polymers. These include polysaccharides such as schizophyllan, glycopolymers, or degradable synthetic polymers [5].

Among the biopolymers, chitosans, a family of cationic and linear polysaccharides derived from chitin, have received increasing attention in biomedical research over the past decade and show an attractive safety profile as well as a range of possibilities for further modifications. Recently, chitosan has emerged as a promising candidate for gene delivery because of biocompatibility, biodegradability, low immunogenicity, low cytotocixity, favorable physicochemical properties and ease of chemical modification [6-9]. The advantage of chitosan-based vectors lies not only in getting away from the cytotocixity problems that are inherent in most synthetic polymeric vehicles, but also in its unique capability of transcellular transport. The presence of positive charges from amine groups enables it to transport plasmid DNA (pDNA) into cells via endocytosis and membrane destability. However, as shown with other polycations/DNA complexes, chitosan/DNA complexes are formed by electrostatic interaction between primary amino groups and phosphate groups, which is strong enough to resist DNA unpacking within cell to a certain degree. Okano, Sato, and Kabanov all reported that the incorporation of hydrophobic moieties could considerably increase the transfection efficiency [10-12]. Wen Guang Liu used N-alkylated chitosan to transfer plasmid-encoding CAT into C2C12 cell lines, and the transfection efficiency is higher than that of chitosan. It is proposed that the higher transfection efficiency of ACS is attributed to the increasing entry into cells facilitated by hydrophobic interactions and easier unpacking of DNA from N-alkylated chitosan carriers due to the weakening of electrostatic attractions between DNA and N-alkylated chitosan [13].

In addition, by theoretical calculations, Kuhn and Levin found that for sufficient hydrophobic amphiphilies, the neutralization or even the inversion of charge of the DNA-amphiphile complexes could achieve with rather low concentration of cationic amphiphile [14].

Ionic liquids, combining good and tunable solubility properties with a negligible vapor pressure and excellent thermal stability, have recently been used for dissolving biological macromolecules including cellulose, wool keratin and silk fibroin that are linked together by intermolecular hydrogen bonds [15-17]. Early data showed that chitosan had a good solubility in 1-butyl-3-methylimidazolium chloride ([BMIM]Cl), and up to $10 \mathrm{wt} \%$ of chitosan can dissolve in this media to form a viscous solution [18].

To the best of our knowledge, to date, research work on the O-alkylation of chitosan on the properties of chitosan/DNA complexes and on the transfection efficiency of chitosan-based vectors is unavailable in the literature. In this work, we synthesized a novel O-alkylated chitosan (OACS) derivatives from dodecanol in ionic liquid [BMIM]Cl in an in-situ reactor, using 
$\mathrm{N}, \mathrm{N}$-carbonyldiimidazole (CDI) as bonding agent, then investigated the solubility of OACS in aqueous acetic acid solution (w/w: $0.1 \%$ ), providing valuable information for the next in-vitro cell transfection. The chemical structure of chitosan (CS) and products were analyzed by FT-IR spectrometer and $\mathrm{x}$-ray diffraction spectrometer.

\section{Experimental}

\subsection{Materials}

Chitosan polymer (50 cP) with a degree of deacetylation (DD) value over $85 \%$ was obtained from XiaMen Sanland Chemicals Co. Ltd. (Xiamen, China). Ionic liquid [BMIM]Cl was prepared as literature [19]. Dodecanol was purchased from J\&K Chemicals Co. Ltd. (Beijing, China) and was freshly distilled before use. N, N'-carbonyldiimidazole (CDI) was supplied by Beijing Chemicals Co. Ltd. (Beijing, China). Chloroform was from Kaixin Fine Chemicals Co. Ltd. (Tianjin, China) and used after dehydration. All other chemicals used were commercially available and used as received.

\subsection{Synthesis of O-Alkylated CS (OACS)}

Herein, an in-situ reactor was elaborately designed for the chitosan alkylation in ionic liquid [BMIM]Cl. The alkylation of CS was performed in the in-situ reactor. Firstly, $0.46 \mathrm{~g}$ of CDI was dissolved by $10 \mathrm{ml}$ of chloroform then added into a three-necked bottle of $50 \mathrm{ml}$. $0.42 \mathrm{~g}$ of dodecanol was dissolved in $10 \mathrm{ml}$ of chloroform and dropped into the three-necked bottle under magnetic stirring and nitrogen atmosphere at $40{ }^{\circ} \mathrm{C}$ for 2 h. Then the chloroform was removed in vacuum in-situ. $0.2 \mathrm{~g}$ of CS was dispersed in $15 \mathrm{~g}$ of ionic liquid [BMIM]Cl. After heated at $80{ }^{\circ} \mathrm{C}$ for $4 \mathrm{~h}$, the liquid was added into the three-necked above bottle and heated to $80{ }^{\circ} \mathrm{C}$ and stirred for 8 h. The reaction mixture was poured into ice water, filtered, and washed with dimethyl ether.

\subsection{Analysis of OACS by FT-IR Spectrometer and X-Ray Diffraction Spectrometer}

The Fourier transform IR (FT-IR) spectra of the samples were recorded with a Fourier transform IR spectrometer (IR Prestige-21, Shimadzu, Japan) in the range of $400-4000 \mathrm{~cm}^{-1}$ in $\mathrm{KBr}$ pellets at ambient temperature. All spectra were recorded with an accumulation of 32 scans and a resolution of $4 \mathrm{~cm}^{-1}$ in the range from 4000 to $400 \mathrm{~cm}^{-1}$. X-ray patterns of powdered samples were obtained using a XRD-6000 X-ray diffract meter (Shimadzu, Japan) with $\mathrm{Cu}$ Ka radiation at $0.15406 \mathrm{~nm}$. The relative intensity was recorded in the scattering range $(2 \theta)$ of $5-50^{\circ}$ with steps of $0.1^{\circ}$ per second.

\section{Results and Discussions}

\subsection{O-Alkylation of CS}

O-alkylation of CS was firstly prepared using CDI as bonding agent. Alkyl was selectively anchored on hydroxyl groups of chitosan without amino group protecting in ionic liquid in an in-situ reactor. Firstly, CDI reacted with dodecanol to produce a reactive intermediate in chloroform. Then the solvent was re- moved under vacuum in-situ to avoid the reaction system exposing into atmosphere, rendering the intermediate deteriorating. The chloroform was necessarily removed lest the solvent lead to insolubilization of CS in [BMIM]Cl, affecting the following reaction of CS with the intermediate in the ionic liquid. To test the solubility of the product, $0.2 \mathrm{mg}$ of OACS was dispersed in $1 \mathrm{ml}$ chloroform, ethanol, water and $0.1 \%(\mathrm{v} / \mathrm{v}) \mathrm{HAc}$ aqueous solution, respectively and observed by naked eyes. It was confirmed that the obtained OACS was hardly dissolved in water, slightly soluble in chloroform and ethanol, easily dissolved in aqueous HAc solution. The protonation of amino groups of CS is responsible for the solubility in HAc aqueous solution. Alkylation of CS improves the solubility of OACS in organic solvent such as chloroform and ethanol. The results show that OACS may be suitable for the cell transfeciton test because of its abundant free amino groups, which are critical in compacting pDNA and delivering genes into cells.

\subsection{IR Characterization of OACS}

Figure 1 shows the IR spectra of unmodified CS and modified chitosan products OACS. The primary amino group of chitosan is recognized by two bands at 1650 and $1544 \mathrm{~cm}^{-1}$ [20]. After alkylation with dodecanol using CDI as bonding reagent in ionic liquid, the amino bands still exist. Two strong bands at $2925 \mathrm{~cm}^{-1}$ and $2856 \mathrm{~cm}^{-1}$, the C-H stretching vibration adsorption peaks, appear, and at $1750 \mathrm{~cm}^{-1}$, the carbonyl group of carbonate band appears. Furthermore, bands at 1461 and 1260 $\mathrm{cm}^{-1}$ also show alkyl and carboxylic groups, respectively. The IR spectrum of OACS (b in figure 1) shows that the dodecanol is anchored selectively on hydroxyl groups of CS, thus the amino groups of CS remain untouched.

Ionic liquid [BMIM]Cl belongs to a class of non-aqueous but polar solvents. This water free solvent is suitable for the reaction using CDI as bonding agent, which is sensitive to water, decomposing into carbon dioxide and imidazole in moist atmosphere. It is composed of cation and anion, displaying acidity, basicity or neutrality, depending on the component. Herein, we found that the ionic liquid solvent can restrain the reaction of amino group, render the alkylation selectively occurring on hydroxyl groups of CS. It is proposed that the nitrogen atoms be in some electron deficiency environment in the solvent,

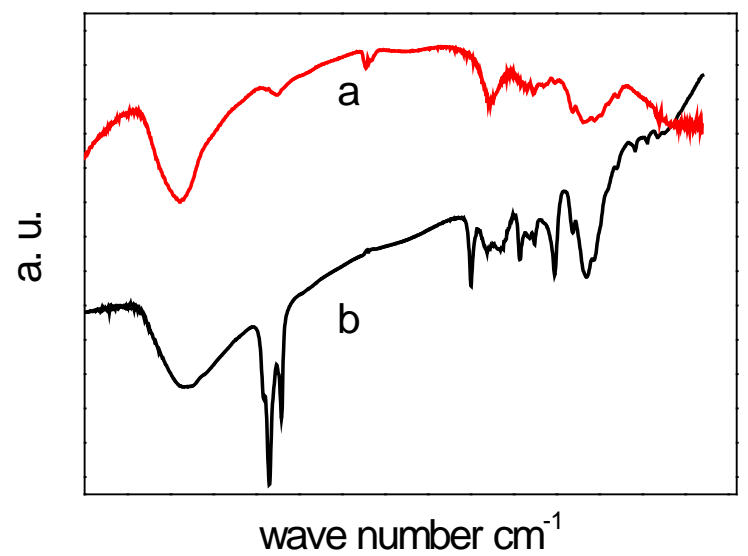

Figure 1. IR spectra of (a) unmodified chitosan, (b) modified chitosan OACS. 
which is harmful for the nucleophilic reaction of amino groups. So the nucleophilic reaction falls into hydroxyl groups of CS, emerging as selectively alkylation of hydroxyl groups without amino groups protecting.

\subsection{XRD Characterization of OACS}

Figure 2 presents XRD patterns of powder CS and power OACS, respectively. In Figure 2a, the original chitosan shows two strong reflections at $2 \theta=10.6$ and $2 \theta=20.4^{\circ}$, which are coincided with the pattern of the form I crystal and the form II crystal [21]. As shown in Figure 2b, the modified chitosan OACS appears two more weak peaks compared with the original chitosan and the crystallinity is $17.2 \%$ while it is $25.4 \%$ for the original chitosan. The results show the amorphous region of OACS increases and the crystalline structure partially changes but maintains the form II crystal region in contrast to the original chitosan. It was reported that the [BMIM]Cl, as an imidazolium based ionic liquids, could depolymerize chitosan effectively under mild conditions [22]. Therefore, the decrease in crystallinity of OACS is most likely to arise from the decomposition of CS to some extent under the reaction conditions.

\section{Conclusions}

O-alkylated chitosan was prepared in ionic liquid [BMIM]Cl in an elaborately designed in-situ reactor, using $\mathrm{N}, \mathrm{N}$-carbonyldiimidazole as bonding agent. The IR spectra characterization results show that the dodecanol is anchored selectively on hydroxyl groups of CS with a new carbonyl functional group, leaving amino groups untouched. It is proposed that the particular properties of the ionic liquid solvent should be responsible for the selective alkylation of hydroxyl groups of CS without protecting amino groups of CS. The XRD patterns of CS and O-alkylated chitosan show that the crystallinity of the latter decreases perhaps due to the docomposition of CS in the ionic liquid. The solubility test of O-alkylated chitosan confirmed that it can be easily dissolved in aqueous HAc solution (w/w: $0.1 \%$ ) because of its abundant free amino groups. The results show that O-alkylated chitosan may be suitable for the following gene delivery test.

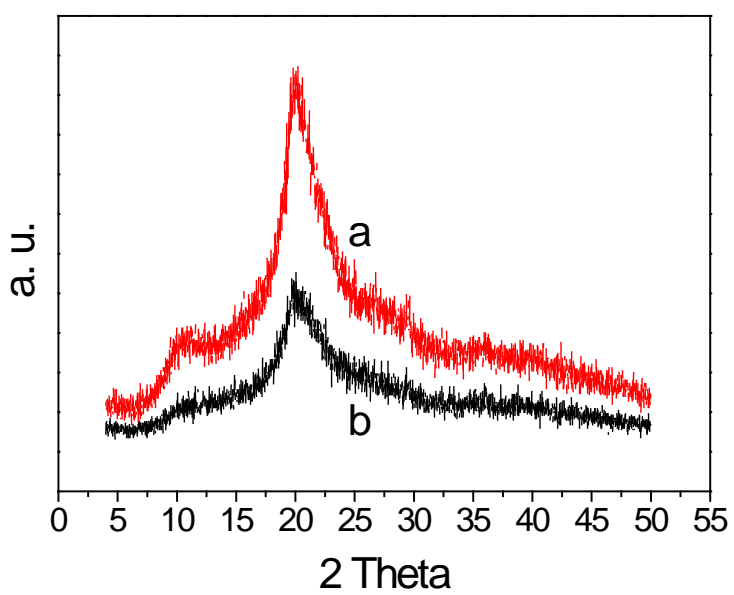

Figure 2. XRD patterns of power samples of (a) unmodified chitosan, (b) modified chitosan OACS.

\section{Acknowledgements}

The authors gratefully acknowledge the financial support from the National Natural Science Foundation of China (21176046, 20876027), and the Fundamental Research Funds for the Central Universities (DC10020103).

\section{REFERENCES}

[1] G. Hellermann, S. S. Mohapatra, "Genetic therapy: on the brink of a new future,” Genet. Vaccines Ther., 2003, vol. 4, pp. 1-9.

[2] M. Breunig, U. Lungwitz, R. Liebl, A. Goepferich, "Breaking up the correlation between efficacy and toxicity for nonviral gene delivery," Proc. Natl. Acad. Sci. U.S.A. 2007, vol. 104, pp. 14454-14459.

[3] H. T. Lv, S. B.Zhang, B. Wang, S. H. Cui, J. Yan, “Toxicity of cationic lipids and cationic polymers in gene delivery,” J. Controlled Release , 2006, vol. 114, pp. 100-109.

[4] D. Luo, M. Saltzman, "Synthetic DNA delivery systems," Nat. Biotechnol., 2000, vol. 18, pp. 33-37.

[5] J. M. Dang, K. W. Leong, "Natural polymers for gene delivery and tissue engineering,” Adv. Drug Delivery Rev., 2006, vol. 58, pp. 487-499.

[6] S. Mansouri, P. Lavigne, K. Corsi, M. Benderdour, E. Beaumont, J. C. Fernandes, "Chitosan/pDNA nanoparticles as non-viral vectors in gene therapy: strategies to improve transfection efficacy,” Eur. J. Pharm. Biopharm., 2004, vol. 57, pp. 1-8.

[7] K. Bowman, K. W. Leong, "Chitosan nanoparticles for oral drug and gene delivery,” Int. J. Nanomed., 2006, vol. 1, pp. 117-128.

[8] K. Y. Lee, “Chitosan and its derivatives for gene delivery,” Macromol.Res., 2007, vol. 15, pp. 195-201.

[9] O. Germershaus, S. R. Mao, J. Sitterberg, U. Bakowsky, T. Kissel, "Gene delivery using chitosan, trimethyl chitosan, or polyethylenglycol graft-trimethyl chitosan block copolymers: establishment of structureactivity relationships in vitro,” J. Controlled Release, 2008, vol. 125, pp. 145-154.

[10] M. Kurisawa, M. Yokoyama, T. Okano, "Transfection efficiency increases by incorporating hydrophobic monomer units into polymeric gene carriers,” J. Controlled Release, 2000, vol. 68, pp. $1-8$.

[11] T. Sato, T. Kawakami, N. Shirakawa, Y. Okahata, "Preparation and characterization of DNA-lipoglutamate complexes,” Bull. Chem. Soc. Jpn., 1995, vol. 68, pp. 2709-2715.

[12] A. V. Kabavov, V. A. Kavanov, "DNA complexes with polycations for the delivery of genetic materials into cells," Bioconjugate Chem., 1995, vol. 6, pp. 7-20.

[13] W. G. Liu, X. Zhang, S. J. Sun, G. J. Sun, K. D. Yao, "N-Alkylated Chitosan as a Potential Nonviral Vector for Gene Transfection,” Bioconjugate Chem., 2003, vol. 14, pp. 782-789.

[14] P. S. Kuhn, Y. Levin, M. C. Barbosa, "Charge inversion in DNA-amphiphile complexes: possible application to gene therapy,” Physica. A, 1999, vol. 274, pp. 8-18.

[15] C. Cuissinat, P. Navard, T. Heinze, "Swelling and dissolution of cellulose, Part V: Cellulose derivatives fibres in aqueous systems and ionic liquids," Cellulose, 2008, vol. 15, pp.75-80.

[16] R. P. Swatloski, S. K. Spear, J. D. Holbrey, R. D. Rogers, “Dissolution of cellulose with ionic liquids," Journal of the American Chemistry Society, 2002, vol. 124, pp. 4974-4975.

[17] H. Zhang, J. Wu, J. Zhang, J. S. He, "1-Allyl-3-methylimidazolium chloride room temperature ionic liquid: A new and powerful nonderivatizing solvent for cellulose,” Macromolecules, 2002, vol. 38, pp. 8272-8277.

[18] H. B. Xie, S. B. Zhang, S. H. Li, "Chitin and chitosan dissolved in ionic liquids as reversible sorbents of CO2.," Green Chemistry, 2002,vol. 8, pp. 630-633.

[19] R. P. Swatloski, S. K. Spear, J. D. Holbrey, R. D. Rogers, “Dis- 
solution of cellulose with ionic liquids,” Journal of the American Chemical Society, 2002, vol. 124, pp. 4974-4975.

[20] H. Susanne, F. Ralf, H. Andreas, S. Frank, "Hydrophobic Chitosan Microparticles: Heterogeneous Phase Reaction of Chitosan with Hydrophobic Carbonyl Reagents," Biomacromolecules, 2007, vol. 8, pp. 2051-2058.
[21] F. Feng, Y. Liu, B. Y. Zhao, K. Hu, "Characterization of half $\mathrm{N}$-acetylated chitosan powders and films," Procedia Engineering, 2012, vol. 27, pp. 718-732.

[22] Z. H. Zhang, C. Z. Li, Q. Wang, Z. B. Zhao, "Efficient hydrolysis of chitosan in ionic liquids Carbohydrate Polymers," Carbohydrate Polymers, 2009, vol. 78, pp. 685-689. 